taneous hemorrhages, dissection of the capsule from the kidney may be either localized or general. An extracapsular hemorrhage may become circumscribed or encysted.

Severe pain in the kidney region, the presence of a tumor-like mass of variable dimensions, and evidence of internal bleeding are important symptoms. The pain is quite variable in character, radiating usually downward, but sometimes toward the shoulder. It may simulate closely the pain of renal colic. At times it is acute. Of great importance is the presence of blood in the urine. In sone cases, jaundice, but not marked, has been noted. In only one case ${ }^{2}$ has the diagnosis been made before operation.

Perirenal hemorrhages, such as these, need prompt operation and the amount of hemorrhage may make the prognosis grave. Whether nephrectomy should be done or not must depend on the conditions.

\section{POSTINFLUENZAL ALOPECIA}

H. H. HAZEN, M.D.

Professor of Dermatology, Georgetown University School of Medicine; Professor of Dermatology, Howard University School of Medicine

WASHINGTON, D. C.

Alopecia following any severe febrile affection is a common occurrence. Morrisey ${ }^{1}$ states that the older literature gives no references to a postinfluenzal alopecia, but Jackson and McMurtry ${ }^{2}$ note that, of ninetyseven private patients suffering from a febrile alopecia, influenza was responsible for nine.

There is still some question as to whether the loss of hair after fevers is due to interference with the nutrition or to a toxin acting directly on the hair papillae, although the former view is more generally held. Most of the writers agree that a preexisting seborrhea makes the loss of hair more certain.

To date I have seen fifty patients suffering from alopecia following influenza. Of these, three were men and forty-seven were women. It is highly probable that women are much more apt to consult a physician, men feeling that the affection is trivial and hardly worth troubling about. Several of the more intelligent barbers have told me that practically all of their customers who had had influenza lost much of their hair. As the epidemic was more severe among the young it is only natural that the alopecia patients were all young. Unquestionably the severity of the disease has much to do with the subsequent loss of hair. One third of my patients had had a severe pneumonia, and only five of the fifty had had fever of less than $102.5 \mathrm{~F}$. On the other hand, I am by no means convinced that a preexisting seborrhea played any great part, for twenty of the fifty stated that they had had practically no dandruff, and the scalps of twenty-two of the fifty were absolutely free from any local trouble, care being taken that in no instance were the scalps washed less than ten days before examination. The average time elapsing from the onset of the influenza to the beginning of the alopecia was nine

2. Baggerd: Zur Kenntnis der Massenblutungenins Nierenlager, Beitr. z. klin. Chir. 91 : $454,1914$.

1. Morrisey, M. J.: Influenza Alopecia, J. Cutan. Dis. 37:177 (March) 1919.

2. Jackson, G. T., and McMurtry, C. W.: A Treatise on Diseases of the Hair, Philadelphia, Lea \& Febiger, 1912. weeks. The shortest time was two weeks, and the longest three months. The amount of hair lost varied greatly. In one case there remained only one tuft of hair on the back of the scalp, and as another extreme, not more than a tenth of the hair was lost. As nearly as can be estimated, largely from the thickness of braids, the average loss of hair was from one third to one half. As a rule only the long hairs fell, and these were almost immediately replaced by short hairs, so it is probable that new hairs simply push out the old hairs that have been damaged. The greatest loss of hair was usually over the anterior and parietal portions of the scalp. The alopecia was diffuse, not patchy, although in two cases there was a tendency toward patchiness. In one instance there was one completely bald area about $1 \frac{1}{2}$ inches in diameter. Only twelve of the patients complained of sensitiveness, either local or general, of the scalp. In fortyone cases both scalp and hair were abnormally dry.

Without exception every patient has developed new hair in a very short space of time; in fact, it would probably be difficult to prevent new hair from coming in.

The treatment is simple: First, the patient must be put in good physical condition and reassured that the prognosis is good. Next, the scalp must be cleansed of any seborrhea. This can usually be done by means of a sulphur ointment. Sufficient vegetable oil must be supplied to the hair to keep it glossy; this is best done by wetting slightly a cloth with the oil, and then rubbing it over separate strands of hair. Sufficient massage should be done twice a day to cause a brisk tingle of the scalp. When the scalp is cleansed of seborrhea, a simple stimulating lotion should be used at night. A dram of salicylic acid to 6 ounces of alcohol or bay rum will work perfectly well. There is not the slightest need for treatment with ultraviolet light or with any form of electrical stimulation. Also there is not the slightest excuse for cutting the hair, unless it be to make the ends even, so that it can be more easily dressed. In all cases a light hat should be worn so as not to interfere with circulation or ventilation.

Postinfluenzal alopecia differs in no wise from any other postfebrile alopecia. In practically all cases the prognosis is excellent. Simple cleansing of the scalp and a stimulating tonic with ordinary hygiene of the sca!p is all the treatment required.

1621 Connecticut Avenue.

Central Council for Infant Welfare-A Central Council for Infant and Child Welfare was inaugurated in London recently. The council consisted of representatives of various infants', children's, mothers' and allied organizations, thirteen in number. The object of the council are to coordinate the work of the various national and local voluntary organizations concerned in the care of motherhood, infancy and childhood by organizing the supply and distribution of funds and workers, both. voluntary and salaried; to establish, supervise and maintain, with the help of government grants and payments by beneficiaries, such residential institutions or to make such other arrangements for the benefit of the mothers and young children as the constituent societies may from time to time find necessary; to provide and promote a standardization of training for social welfare workers in connection with motherhood, infancy and childhood, and to raise the status and remuneration of the workers. The chairman of the council is Sir Arthur Stanley, and the secretary is Miss Wilson; the offices for the time being will be at 83, PaH Mall.-Medical Officer 21:122, 1919. 